\title{
Graft-induced Changes in MicroRNA Expression Patterns in Citrus Leaf Petioles
}

\author{
R. Tzarfati ${ }^{1}$, S. Ben-Dor ${ }^{2}$, I. Sela ${ }^{1}$ and E.E. Goldschmidt* ${ }^{*}$ \\ ${ }^{1}$ Institute of Plant Sciences and Genetics in Agriculture, The Robert H. Smith Faculty of Agriculture, Food and \\ Environment, The Hebrew University of Jerusalem, Rehovot 76100, Israel; ${ }^{2}$ Department of Biological Services, The \\ Weizmann Institute of Science, Rehovot 76100, Israel
}

\begin{abstract}
Grafting is an important, widely used plant propagation technique but its physiological effects are as yet insufficiently understood. Recent studies indicate that movement of proteins and small RNAs through the graft union might be involved. MicroRNAs are known to play a significant role in regulation of higher plants' developmental and metabolic traits. Extending this logic, we hypothesize that changes in activity of specific microRNAs are one of the mechanisms involved in physiological effects of grafting. The objective of the present study was to test this hypothesis. We determined the expression of a broad range of microRNAs in Citrus leaf petioles, as affected by grafting. Four stock/scion combinations ('Merav' mandarin and 'Star Ruby' grapefruit scions X 'Troyer' citrange and 'Volkamer' lemon rootstocks), rootstock auto-grafts and plants of the variety used as rootstock (= non-grafted) were examined. Grafting caused a dramatic reduction in the expression of the major microRNAs, miR156 (and miR157), which appear to be associated with reduction of juvenility in perennial woody plants. This effect was strongest in hetero-grafts but evident also in auto-grafts. Expression of miR894 also declined upon grafting. Differences in the expression of miR397 were found among grafted scion cultivars, while in non-grafted rootstocks expression of miR397 was barely detectable. Bioinformatic analysis confirmed the presence of miR397 in the citrus genome, validated its sequence and demonstrated its ability to form a stem loop. The differences in miR397 expression might be related to specific copper and other micronutrient requirements of citrus stock-scion combinations.Thus, our results support the hypothesis, indicating the involvement of specific microRNAs in engendering physiological effects of grafting in Citrus. The precise, underlying mechanism needs to be elucidated.
\end{abstract}

Keywords: Citrus, grafting effects, juvenility, rootstock, scion.

\section{INTRODUCTION}

Grafting of plants has been practiced throughout the ages [1] and is used widely even today in culture of fruit trees, ornamentals [e.g. roses] and as of recently, vegetables [2]. A major advantage of propagation by grafting is the marked shortening of the juvenile phase, which in tree seedlings may last several years or, in forest trees, up to several decades $[1,3]$. The interactions between rootstocks and scions are diverse, complex and as yet not fully understood. While anatomical aspects, supply of water, mineral nutrients and transport of plant hormones have been implicated, recent work demonstrated cross-graft transfer of specific proteins [4] and RNA-induced gene silencing [5,6]. Thus, molecular mechanisms are currently assumed to be involved in physiological effects of grafting and in mediation of stock/scion interactions. MicroRNAs are currently known to be involved in regulation of a large number of developmental and metabolic plant traits [7-9]. Evidence for graft transmission of specific microRNAs has been

*Address correespondence to this author at the Institute of Plant Sciences and Genetics in Agriculture, The Robert H. Smith Faculty of Agriculture, Food and Environment, The Hebrew University of Jerusalem, Rehovot 76100, Israel; Tel: +972-8-9489353; Fax: +972-8-9489899;

E-mail: eli.goldsmit@mail.huji.ac.il accumulating in recent years, in various plant species [1013].

Although grafted plants have been used extensively as experimental systems in small RNA research, a general role for microRNAs in physiological effects of grafting has not been hypothesized, to the best of our knowledge. The objective of the present study was to test this hypothesis in citrus and search for graft-related differences in expression of specific microRNAs. We used young, potted citrus trees as our experimental system, examining several grafted stock/scion combinations, rootstock auto-grafts and nongrafted controls Fig. (1). Petioles of fully expanded leaves were used as a source of RNA, the rationale being that petioles consist predominantly of vascular tissues in which transportable compounds may be abundant. Unlike studies that concentrated on a small number of presumably relevant microRNAs, we adopted a screening strategy, examining the expression of a broad spectrum of microRNAs.

Here we show that grafting induced changes in the expression pattern of several ubiquitous, physiologically significant microRNA species in citrus leaf petioles. Differences in the expression pattern of smaller, specific microRNAs among citrus' stock/scion combinations were also demonstrated, thereby supporting our hypothesis. 


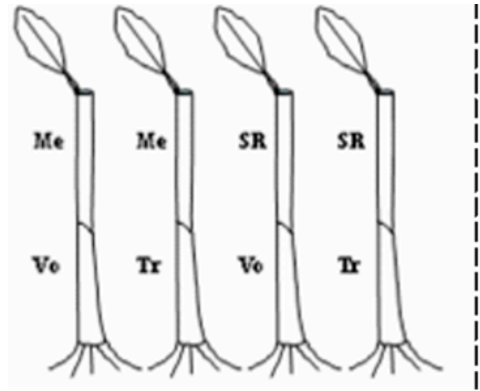

Hetero-Grafts

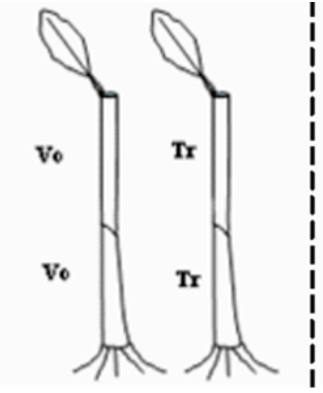

Auto-Grafts

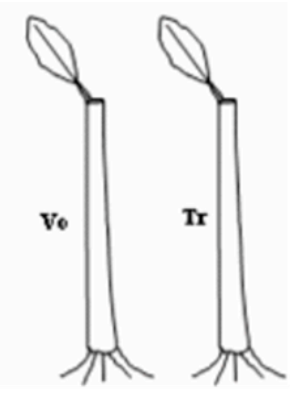

Non-Grafts

Fig. (1). Schematic illustration of the 4 hetero-graft combinations, auto-grafts, and non-grafted control plants of the same variety(=nongrafts) used in the present study.

Table 1. Citrus cultivars used in the present study.

\begin{tabular}{|l|l|l|l|}
\hline Horticultural use & Scientific name & Abbreviation & Cultivar \\
\hline \hline scion & Citrus reticulata Blanco & $\mathrm{Me}$ & 'Merav' mandarin \\
\hline scion & Citrus paradisi Macf. & $\mathrm{SR}$ & 'Star Ruby' grapefruit \\
\hline rootstock & Citrus volkameriana & $\mathrm{Vo}$ & 'Volkamer' lemon \\
\hline rootstock & Poncirus trifoliata X Citrus sinensis & $\mathrm{Tr}$ & 'Troyer' citrange \\
\hline
\end{tabular}

\section{MATERIAL AND METHODS}

\section{Plant Material and RNA Isolation}

Grafted and non-grafted, container grown, 2 year old citrus trees were obtained from 'Bney Dror' Nurseries, Israel, courtesy of Mr. Shuli Rosen. Grafting was performed during spring of the second year; the graft union was smooth and healthy. Four stock/scion hetero-graft combinations (2 scions X 2 rootstocks), 2 rootstock auto-grafts and 2 nongrafted controls were tested Fig. (1). The citrus cultivars used (and their abbreviations) are listed in Table $\mathbf{1}$. Individual seedlings served as replicates.

Petioles of fully expanded leaves were used for RNA analysis. Leaf petioles were collected during mid-summer (July-August) from actively growing trees by late morning $(9-10 \mathrm{am})$, when leaf activity was already on. The effect of wounding was also examined. The wounding treatment consisted of scratching $20 \mathrm{~cm}$ along stems of rootstock seedlings $48 \mathrm{~h}$ before the sampling of leaf petioles. Fully expanded leaves were plucked using sterile gloves, the petioles removed and instantaneously frozen in liquid nitrogen. Total RNAs were extracted from petiole tissue using a 'Norgen' biotek corporation Plant/Fungal Total RNA Isolation Kit 50R 25800.

\section{MicroRNA Microarray Assay}

Microarray assay was performed using a service provider (LC Sciences, Houston TX, USA) according to their protocol and statistical outlay. The custom upraflo $^{\mathrm{TM}}$ microfluidic chip contained plant miRNAs of release version 13.0 (http://microrna.sanger.ac.uk).

\section{Bioinformatics}

Database searches were run at HarvEST (http://138.23.191.145/blast/index.html) against version 1.25 of HarvEST Citrus and the JGI Citrus genome database (citrus sinensis), using the default parameters with the exception of E-value, which was raised to 1000 due to the short input sequence length. Searches were also performed at NCBI blast (http://blast.ncbi.nlm.nih.gov/), against both NR and est_other databases, with default parameters, with exceptions of E-value of 1000, the blastn algorithm, and organism limited to citrus (taxid:2706). Alignments were performed with ClustalW 2.012 [14] and RNA secondary structure was predicted with both Mfold [15] and the Vienna package [16].

\section{TaqMan miRNA Assay}

For validation of miRNA microarray results, TaqMan miRNA assays (Applied Biosystems) were used according to the manufacturer's protocol [17]. Levels of the citrus miR397, a perfect match to miR397a, were normalized to the miR164a control; miR164 a was used as a normalizing gene since members of this family were highly expressed, with least variation among treatments (Suppl. Table 1). All qRTPCR reactions were performed on ABI7300 machine. Results are presented as means and standard deviation of two biological replicates.

\section{RESULTS}

The miRNAs identified through microarray in the citrus cultivars examined are listed in Suppl. Table 1. A total of 95 miRNAs belonging to 35 families were detected.The expression rates of nine ubiquitous microRNAs in petioles of the two scion cultivars, 'Merav' mandarin and 'Star Ruby' grapefruit, grafted on 'Volkamer' and 'Troyer' rootstocks and the non-grafted controls were compared in the experiment presented in Fig. (2). A significant difference between the grafted and non-grafted petioles appeared with miR156 and miR157. The expression of these miRs was much higher in the non-grafted rootstocks than in either grafted scion cultivar, in 'Volkamer' more than in 'Troyer'. A similar, although less striking trend appeared also with miR894, which expressed more strongly in the non-grafted rootstocks than in the grafted scions Fig. (2). Strong 


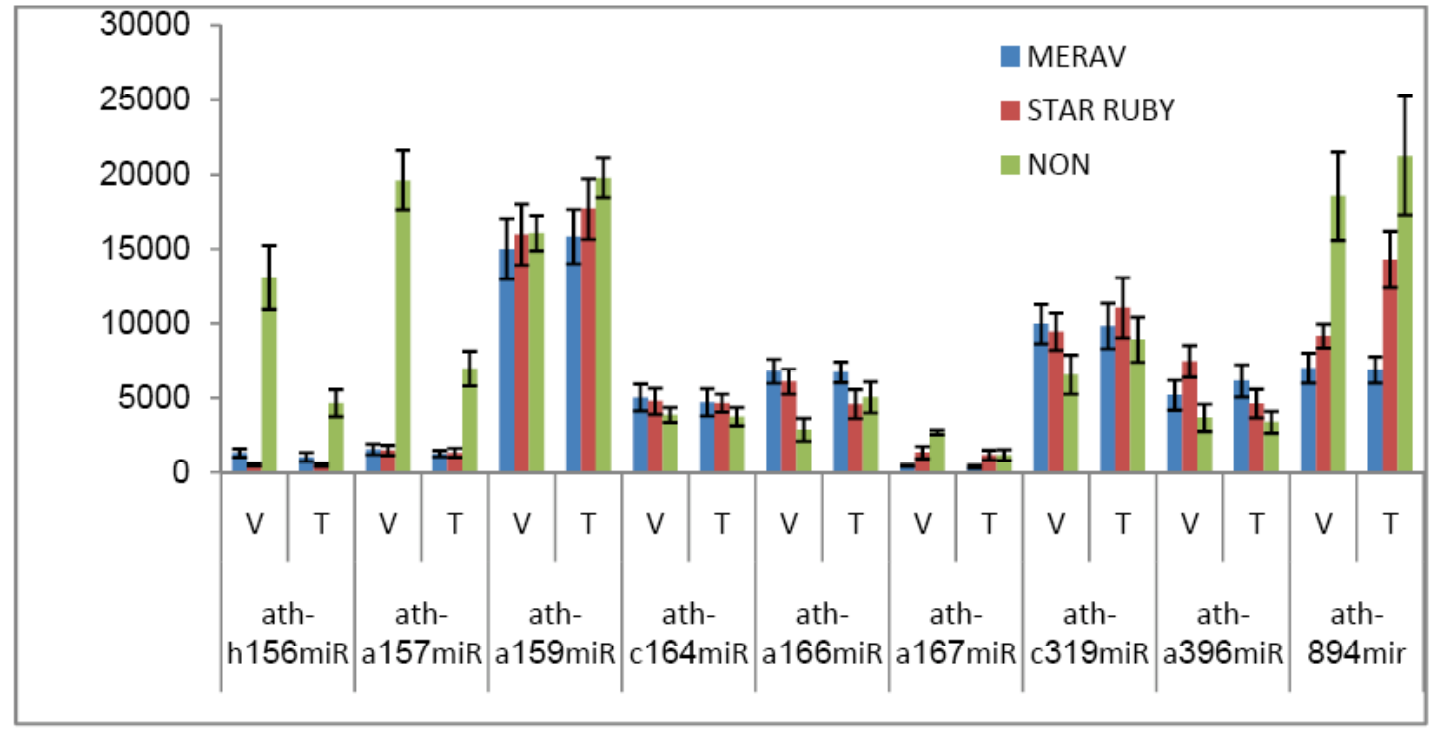

Fig. (2). Relative microarray expression pattern of 9 major micro RNA families in citrus petioles of 'Merav' and 'Star Ruby' grafted on 'Volkamer'[Vo] and 'Troyer'[Tr] rootstocks and the non-grafted controls[NON]. Averages from 6 replicates $+/-$ SD. Identical results obtained for each family with other copies; e.g. for miR156 with ath-h, smo-b, pta-a, pta-b.

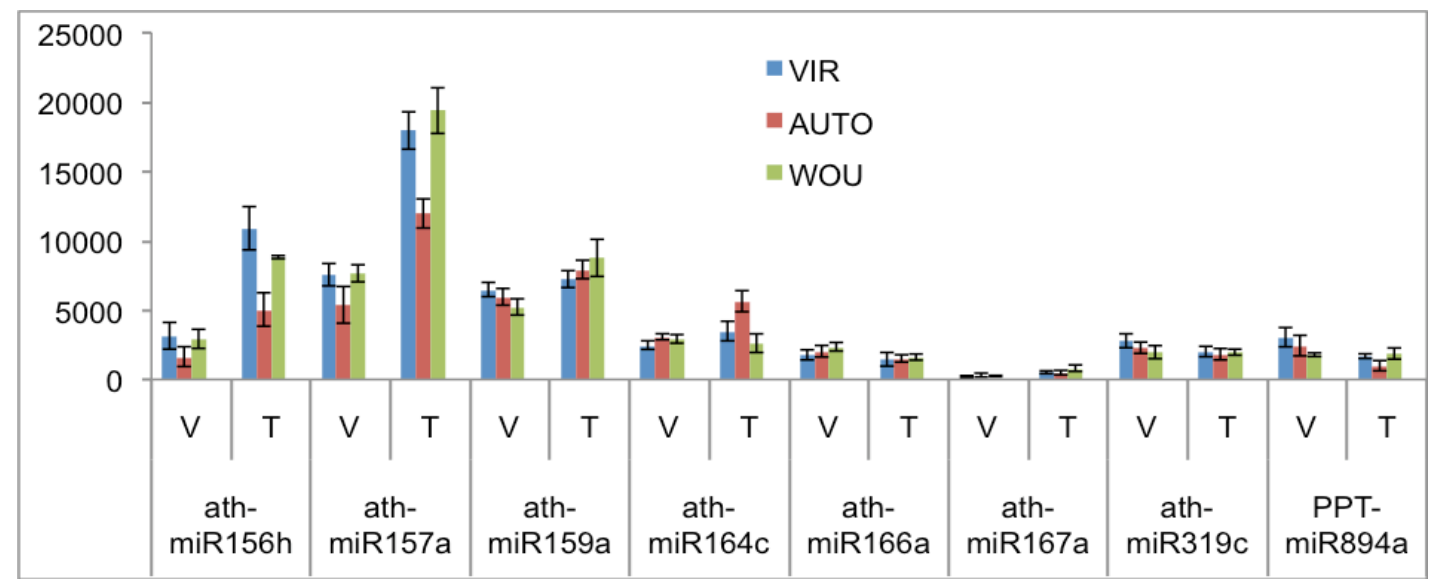

Fig. (3). Relative microarray expression pattern of 8 major micro RNA families in citrus petioles of non-grafted [NON], auto-grafted [AUTO], and wounded [WOU] 'Volkamer' [Vo] and 'Troyer' $[\mathrm{Tr}]$ plants. Averages from 3 replicates +/-SD. Identical results obtained for each family with other copies; as in Fig. (2).

expression of miRs 156, 157, and 894 appeared also in a separate comparison of the non-grafted rootstocks (data not shown). With other miRs (miRs 159, 164, 166, 167, 319, $396)$ the expression rates of the grafted and non-grafted were similar and did not reveal striking differences. Although the expression of miR172 in our leaf petiole system was quite low, the activity was still somewhat higher in the grafted scions than in the non-grafted plants (data not shown).

In another experiment we compared the microRNA expression patterns in petioles of non-grafted 'Volkamer' and 'Troyer' plants with auto-grafts of the same rootstock cultivars; the effect of wounding was also tested in this experiment Fig. (3). The expression of miR156 and miR157 was considerably higher in the non-grafted plants than in the corresponding auto-grafts. Wounding of the non-grafted plants did not seem to affect the expression of miRs 156, 157. Neither auto-grafts nor wounding seemed to influence the expression of other major miRs Fig. (3), or any other miRs.

The comparison between the 4 hetero-graft, stock/scion combinations was intended as a probe for apparent rootstock effects. The microarray analysis clusters (not shown) revealed 3 miRNA families which were expressed stronger in both 'Merav' and 'Star Ruby' scions when grafted on 'Troyer', than when grafted on 'Volkamer' rootstock ; miR397, miR398, and miR408. Among those, miR397 provided the most striking differences between grafts on 'Volkamer' and grafts on 'Troyer'. Fig. (4) demonstrates the differences obtained in the microarray analysis with 5 members of the miR397 family; the expression on 'Troyer' was consistently higher than on 'Volkamer' rootstock. However, the difference in miR397 expression between grafts on 'Troyer' and 'Volkamer' was more prominent with 'Star Ruby' than with 'Merav' scion Fig. (4). Not of lesser significance is the finding that members of the miR397 


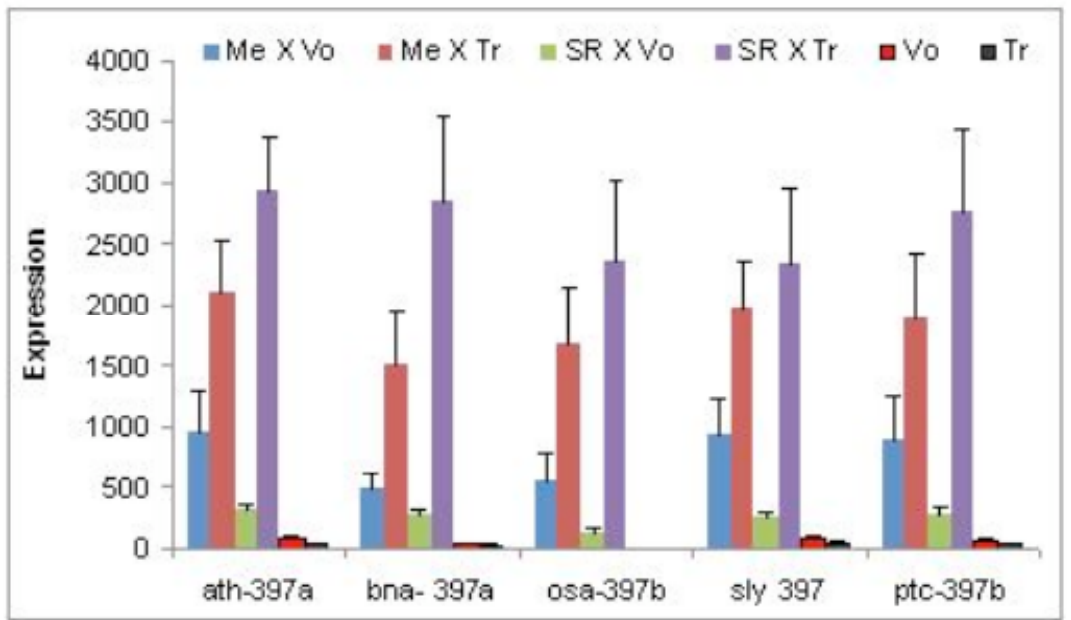

Fig. (4). Relative microarray expression pattern of miR397 family members in citrus petioles of the 4 hetero-grafts and 2 non-grafted control plants. Averages from 6 biological replicates, each consisting of 2 technical replicates \pm SD.

citrus-miR397a
ath-miR397a
bdi-miR397
bna-miR397a
bna-miR397b
osa-miR397a
ptc-miR397a
vvi-miR397a
vvi-miR397b
ath-miR397b
osa-miR397b
ptc-miR397b
sly-miR397

UCAUUGAGUGCAGCGUUGAUG-
UCAUUGAGUGCAGCGUUGAUG-
UCAUUGAGUGCAGCGUUGAUG-
UCAUUGAGUGCAGCGUUGAUGU
UCAUUGAGUGCAGCGUUGAUGU
UCAUUGAGUGCAGCGUUGAUG-
UCAUUGAGUGCAGCGUUGAUG-
UCAUUGAGUGCAGCGUUGAUG-
UCAUUGAGUGCAGCGUUGAUG-
UCAUUGAGUGCAUCGUUGAUG-
UUAUUGAGUGCAGCGUUGAUG-
CCAUUGAGUGCAGCGUUGAUG-
--AUUGAGUGCAGCGUUGAUGA

Fig. (5). Alignment of miR-397a from various plant species. Plant miR-397 found in miRBase (www.mirbase.org) were taken as input to the alignment. Identical sequences from the same species were removed. Differences are highlighted in red.

family, which showed consistent differences between the scions grafted on 'Volkamer' and 'Troyer' rootstocks, did not show such differences between the non-grafted control plants Fig. (4). In fact, the miR397 family was either weakly expressed (ath miR397a, b) or completely undetectable (bna miR397a, osa miR397b, ptc miR397c, sly miR397) in the non-grafted plants (Suppl. Table 1, and Fig. (4). The lack of detectable miR397 expression in the petioles of non-grafted 'Volkamer' and 'Troyer' plants was reconfirmed in a separate experiment (data not shown).

Thus, miR397 appeared to be the best candidate for further validation through qRT PCR. However, as its presence in citrus was based previously on prediction from Arabidopsis [18], we performed bioinformatic analyses to confirm its presence in citrus and validate the exact sequence. Sequence based searches with the arabidopsis miR397a in citrus ESTs and mRNAs in NCBI and ESTs at HarvEST did not yield any results. However, when we searched against the JGI Citrus genome database, two perfect matches were found (BUZZ68439.b1, and BUZZ65818.g1) The two hits had a perfect overlap of $466 \mathrm{bp}$, including the miR397a sequence. The putative miR sequence was a perfect match to miR397a from arabidopsis, bdi, bna (which has an additional terminal T), osa, ptc, and vvi Fig. (5). We tested the ability of the genomic DNA in this region to form a stem-loop, required for the processing of miR. We took both $100 \mathrm{bp}$ up and downstream of the $\mathrm{miR}$ and the region equivalent to the stem-loop of arabidopsis 397a (110 bp total) and both formed stem-loops, with the miR in a stem region Fig. (6). miR397 was subsequently sequenced independently by Song et al. [9], confirming what we had found in our analyses.

The results of the qRT PCR assays confirmed the stronger activity of miR397 in both scions on 'Troyer' than on 'Volkamer' rootstock Figs. (7A and 7B). In the qRT PCR as well, the quantitative difference between the rootstocks was more prominent with 'Star Ruby' than with 'Merav'scion.

\section{DISCUSSION}

The growing interest in the role of small RNAs has resulted in a large number of publications that describe the microRNA population of various crop plants, including citrus [18-20]. Yet, studies relating to the physiological roles 
of microRNAs are still scarce, and nothing of this kind has been reported so far for Citrus.

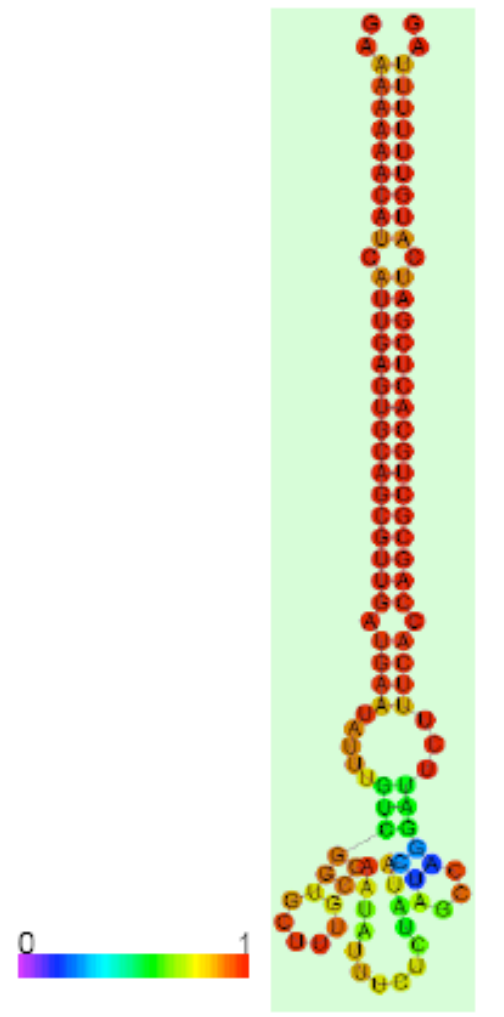

Fig. (6). Predicted folding of the citrus miR397a. The sequence equivalent to the published arabidposis stem-loop was taken as input to RNAfold (Gruber et al.) [16]. The minimum free energy of this conformation is $-43.30 \mathrm{kcal} / \mathrm{mol}$. The drawing is colored according to the base-pairing potential, with red meaning almost definitely in a pair. Note that the region of the stem is very significantly paired.

Grafting has been used extensively in experimental plant research, in classical studies of floral induction [21] as well as in recent investigations [22]. Grafting has played an important role in studies of gene silencing [5], mainly as a

A

miR-397/miR-164

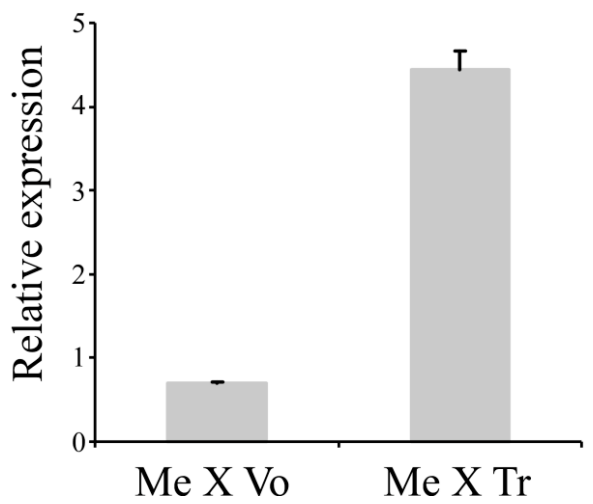

probe for the long distance movement of various kinds of small RNA [12]. Graft transmission of the tuberization inducer miR172 has been demonstrated in potato [11] and in Nicotiana benthamiana [23]. The role of graft-transmissible MicroRNA signaling has been demonstrated with P [24], S, $\mathrm{Cu}$ and $\mathrm{Fe}$ mineral starvation [10].

In the present study we focused on the possible involvement of microRNAs in physiological effects of grafting in young citrus trees. We had initially intended to use shoot sap, but the difficulties in obtaining substantial amounts of sap from citrus shoots [25] turned us to the use of mature, non-growing leaf petioles which consist mostly of vascular elements. Since the xylem appears to be devoid of RNAs [26] petiole extracts are presumably enriched with phloem sap RNA components. The micro RNA population of phloem tissue and phloem sap has been reported for Cucumis, Lupinus [27] Brassica [26], and Malus [13]).

In the study of grafting physiology it seems important to distinguish between primary, major effects, which concern any grafted plant, and secondary, minor effects which reveal differences between specific rootstock/scion combinations. Most horticultural studies have concentrated up to now on the latter, secondary effects, and paid little attention to the primary effects. In the present experimental study both types of effects became apparent.

In the discussion of our results it seems appropriate to begin with the data of the primary, major microRNAs Figs. ( 2 and 3). The most intriguing result is the reduced expression of miR156 and miR157 in petioles of all grafted combinations as compared with their respective non-grafted control plants. Extreme reduction was obtained in grafted scion cultivars Fig. (2) and considerable reduction appeared also in the auto-grafts of both rootstock cultivars Fig. (3). The significance of these findings becomes apparent in view of recent work on the vegetative phase change in annuals and trees [28, 29]. According to Wang et al. [29] the juvenile-toadult transition in tree species involves a marked decline in the activity of miR156. Reduction of juvenility is one of the most significant effects of grafting in trees [1,3]. Thus, the observed reduction of miR156, and the closely related miR157 [30] activities in grafted citrus Figs. (2 and 3) fits

B
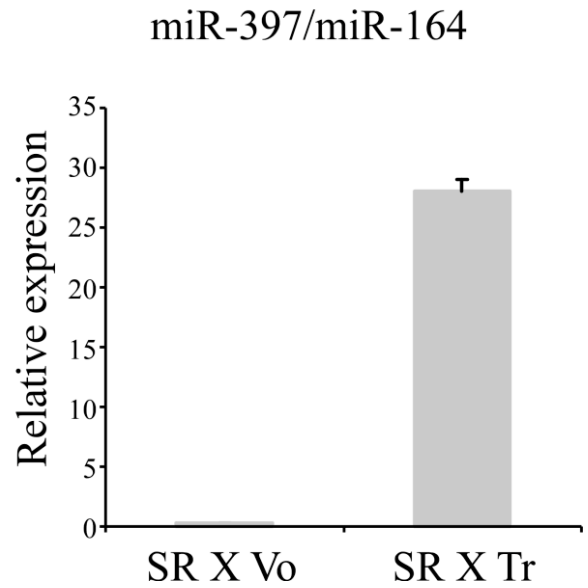

Fig. (7). Comparison of qRT- PCR activity of miR397 in petioles of each scion (Me, SR) on the 2 rootstocks( Vo, Tr ); miR164 was used as the normalizing gene (see Methods). Averages from 2 replicates, each consisting of 3 technical replicates \pm SD. 
very well into the presumed role of these micro RNAs in the juvenile-to-adult transition in trees. It is conceivable that auto-grafts reveal a more moderate reduction of juvenility than hetero-grafts(that represent an interaction between two plant genomes); which explains why the reduction in the expression of miRs 156, 157 was more pronounced in hetergrafts Fig. (2) than in auto-grafts Fig. (3). According to Wang et al. [29], the acquisition of the adult phase also involves a gradual increase in the expression of miR172. Slightly higher expression of miR172 in petioles of the grafted scions, as compared with non-grafted control plants, has been obtained in the present study as well (data not shown).

The search for differential expression of a specific micro RNAs between distinct rootstock/scion combinations has led to the identification of miR397, which expressed more strongly, in both scions, when grafted on 'Troyer' than when grafted on 'Volkamer' Figs. (4 and 7). The presence of miR397 in citrus has recently been demonstrated by Song et al. [19], and its targeting of IRX12 was confirmed. Expression of the miR397 family is induced by various stress treatments [31]. Members of miR397 target copper binding to proteins in Arabidopsis [32] and play a role in plant heavy metal homeostasis [33]. Growth under copper deficiency is known to induce a number of physiological responses, including the expression of miRs 397, 398, 408 [34], which were also differentially expressed in the present study. In Brassica napus copper deficiency led to a more than four-fold increase of miR397 expression in phloem sap [10]. Involvement of microRNA signaling in additional mineral nutrition elements has been demonstrated in Brassica [10,24]. The observed differential expression of miR397 in citrus' stock/scion combinations Figs. (4 and 7) might explain subtle differences in copper and other micronutrient requirements between citrus rootstocks and graft combinations [35-37].

All in all, the results of the present study lend support the hypothesis that microRNA expression plays a role in the mediation of both primary and secondary effects of grafting. Extension of these studies to other grafted plant systems and elucidation of the precise physiological mechanisms involved requires further research.

\section{CONFLICT OF INTEREST}

The authors confirm that this article content has no conflicts of interest.

\section{ACKNOWLEDGEMENTS}

We thank the Israeli Citrus Marketing Board and Dr Y. Erner for financial support of this project and the Rokach Foundation for a financial award to Mr. Raanan Tzarfati. Drs. T. Arazi and Y. Peretz helped during the early stages of the project. We thank Dr. Y. Buganim for advice and discussions during preparation of the manuscript and Ms. Y. Scherbina for help with the illustrations.

\section{SUPPLEMENTARY MATERIAL}

Supplementary material is available on the publishers Web site along with the published article.

\section{REFERENCES}

[1] Mudge K, Janick J, Scofield S, Goldschmidt, EE. A history of grafting. Hortic Rev 2009; 35: 437- 93.

[2] Davis AR, Perkins-Veazie P, Sakata Y, et al. Cucurbit grafting. Crit Rev Plant Sci 2008; 27: 50-74.

[3] Wareing PF. Problems of juvenility and flowering in trees. J Linn Soc Lond (Botany) 1959; 56: 282-9.

[4] Pina A, Errea PP. A review of new advances in mechanism of graft compatibility-incompatibility. Sci Hortic 2005; 106: 1-11.

[5] Palauqui JC, Elmayan T, Polien JM, Vaucheret H. Systemic acquired silencing: transgene-specific post-transcriptional silencing is transmitted by grafting from silenced stocks to non-silenced scions. EMBO J 1997; 16: 4738-45.

[6] Shaharuddin NA, Han Y, Li H, Grierson D. The mechanism of graft transmission of sense and antisense gene silencing in tomato plants. FEBS Lett 2006; 580: 6579-86.

[7] Jones-Rhodes MW, Bartel DP, Barterl B. MicroRNAs and their regulatory roles in plants. Ann Rev Plant Biol 2006; 57: 19-53.

[8] Meng Y, Shao C, Chen M. Toward microRNA-mediated gene regulatory networks in plants. Brief Bioinform 2011; 12: 645-9.

[9] Zhang B, Pan X, Cobb GP, Anderson TA. Plant microRNA: a small regulatory molecule with big impact. Dev Biol 2006; 289: 316.

[10] Buhtz A, Pieritz J, Springer F, Kehr J. Phloem small RNAs, nutrient stress responses, and systemic mobility. BMC Plant Biol 2010; 10: 64-76.

[11] Martin A, Adam H, Diaz-Mendoza M, Zurczak M, GonzalezSchain ND, Suarez-Lopez P. Graft transmissible induction of potato tuberization by the microRNA miR172. Development 2009; 136: $2873-81$.

[12] Melnyck CW, Molnar A, Baulcombe DC. Intercellular and systemic movement of RNA silencing signals. EMBO J 2011; 30: 3553-63.

[13] Varkonyi-Gasic E, Gould N, Sandanayaka M, Sutherland P, Mac Diarmid RM. Characterisation of microRNAs from apple (Malus domestica 'Royal Gala') vascular tissue and phloem sap. BMC Plant Biol 2010; 10: 159-74.

[14] Larkin MA, Blackshields G, Brown NP, et al. Clustal W and Clustal X version 2.0. Bioinformatics 2007; 23: 2947-8.

[15] Zuker M. Mfold web server for nucleic acid folding and hybridization prediction. Nucleic Acids Res 2003; 31: 3406-15.

[16] Gruber AR, Lorenz R, Bernhart SH, Neuböck R, Hofacker IL. The Vienna RNA Websuite. Nucleic Acids Res 2008; 36(suppl 2) W70W74.

[17] Kahl G, Meksem K, Eds. The Handbook of Plant Functional Genomics. Weinheim, Germany: Wiley-VCH Verlag GmbH \& Co 2008.

[18] Song C, Fang J, Li X, Liu H, Chao CT. Identification and characterization of 27 conserved micro RNAs in citrus. Planta 2009; 230: 671-85

[19] Song C, Wang C, Zhang C, et al. Deep sequencing discovery of novel and conserved microRNAs in trifoliate orange (Citrus trifoliata). BMC Genomics 2010; 11: 431-43

[20] Wu XM, Liu MY, Guo WW. Identification and characterization of microRNAs from citrus expressed sequence tags. Tree Genet Genomes 2011; 7:117-33.

[21] Evans LT, Ed. The Induction of Flowering: Some Case Histories. Mac Millan of Australia 1969.

[22] Wang YQ. Plant grafting and its application in biological research. Chin Sci Bull 2011; 56: 3511-7

[23] Kasay A, Kanehira A, Harada T. miR 172 can move long distances in Nicotiana benthamiana. Open Plant Sci J 2008; 4: 1-6.

[24] Pant BD, Buhtz A, Kehr J, Scheible WR. MicroRNA399 is a longdistance signal for the regulation of plant phosphate homeostasis. Plant J 2008; 53: 731-8

[25] Saidha T, Goldschmidt EE, Monselise SP. Endogenous growth regulators in tracheal sap of citrus. Hortic Sci 1983; 18: 231-32.

[26] Buhtz A, Springer F, Chappel IL, Baulcombe DC, Kehr J. Identification and characterization of small RNAs from the phloem of Brassica napus. Plant J 2008; 53: 739-49.

[27] Yoo B, Kragler F, Varkonyi-Gasic E, et al. A systemic small RNA signaling system in plants. Plant Cell 2004;16: 1979-2000.

[28] Poethig RS. Small RNAs and developmental timing in plants. Curr Opin Genet Dev 2009; 19: 374-8.

[29] Wang JW, Park MY, Wang LJ, et al. MiRNA control of vegetative phase change in trees. PLoS Genet 2011; 7: 1-8. 
[30] Rhodes MW, Reinhart BJ, Lim LP, Burge CB, Bartel B, Bartel D. Prediction of plant microRNA targets. Cell 2002; 110: 513-20.

[31] Sunkar R, Zhu JK. Novel and stress-regulated microRNAs and other small RNAs from Arabidopsis. Plant Cell 2004; 16: 2001-19.

[32] Abdel-Ghany SH, Pilon M. MicroRNA-mediated systemic downregulation of copper protein expression in response to low copper availability in Arabidopsis. J Biol Chem 2009; 283: 15932-45.

[33] Ding YF, Cheng Z. The role of microRNAs in copper and cadmium homeostasis. Biochem Biophys Res Commun 2009; 386: 6-10.

[34] Yamasaki H, Makoto H, Fukazawa M, Kobayashi Y, Shikanai T. SQUAMOSA promoter binding protein-like7 is a central regulator for copper homeostasis in Arabidopsis. Plant Cell 2009; 21: 34761.

[35] Iqbal S, Chaudhary MI, Anjum MA. Effect of various rootstocks on leaf mineral composition and productivity of Kinnow mandarin. Int J Agric Biol 1999; 1: 91-3.

[36] Toplu C, Kaplankiran T, Demirkeser H, Yildiz E. The effect of citrus rootstocks on Valencia Late and Rhode Island Valencia oranges for some plant nutrient elements. Afr J Biotechnol 2008; 7 : $4441-5$.

[37] Wutscher HK. Rootstocks and mineral nutrition of citrus. In: Jackson LK, Krezdorn AH, Soule J, Eds. Proc First Intern Citrus Short Course, Univ of Florida Gainesville 1973; pp.97-113

(C) Tzarfati et al.; Licensee Bentham Open.

This is an open access article licensed under the terms of the Creative Commons Attribution Non-Commercial License (http://creativecommons.org/licenses/by-nc/3.0/) which permits unrestricted, non-commercial use, distribution and reproduction in any medium, provided the work is properly cited. 